\title{
A New Approach to Automated Retinal Vessel Segmentation Using Multiscale Analysis
}

\author{
Qin Li, Jane You, Lei Zhang, and David Zhang \\ Department of Computing \\ The Hong Kong Polytechnic University \\ KLN, Hong Kong \\ csqinli@comp.polyu.edu.hk
}

\begin{abstract}
Computer based analysis for automated segmentation of blood vessels in retinal images will help eye care specialists screen larger populations for vessel abnormalities. However, automated retinal segmentation is complicated by the fact that the width of retinal vessels can vary from very large to very small, and that the local contrast of vessels is unstable, especially in unhealthy ocular fundus. We propose a novel method that takes these facts into account. Our method includes a multiscale analytical scheme using Gabor filters and scale production, and a threshold probing technique utilizing the features of retinal vessel network. Our method is good for detecting large and small vessels concurrently. It also offers an efficient way to denoise and enhance the responses of line filters, allowing the detection of vessels with low local contrast.
\end{abstract}

\section{Introduction}

Images of the ocular fundus tell us about retinal, ophthalmic, and even systemic diseases such as diabetes, hypertension, and arteriosclerosis. A central feature in such diagnoses is the appearance of blood vessels in ocular fundus. The development of an efficient and effective computer based approach to the automated segmentation of blood vessels in retinal images would allow eye care specialists to screen larger populations for vessel abnormalities. However, automated retinal segmentation is complicated by the fact that the width of retinal vessels can vary from very large to very small, and that the local contrast of vessels is unstable, especially in unhealthy ocular fundus.

Previous vessel segmentation methods were either window-based [1] [2] [3] or tracking based [4] [5] [6] [7]. Window-based methods explore the properties of a pixel's surrounding window and emphasize those pixels whose surrounding window matches a given model. Most window-based methods implement classical line detection techniques on vessel fields. In [1], the cross section of a vessel is modeled by a Gaussian shaped curve and the matched filters of 12 directions are used to emphasize vessels. Tracking-based methods utilize a vessel profile model, starting from a number of initial points and incrementally tracing a path that best matches the profile

\author{
Prabir Bhattacharya \\ Institute for Information Systems Engineering \\ Concordia University \\ Quebec, Canada \\ prabir@ciise.concordia.ca
}

model. In [4], the tracking starts from the papilla, and then is followed with the consideration of the continuities of position, curvature, diameter, and density. In [6], the tracking is improved using a fuzzy model of a vessel profile. In [8], the window-based and tracking-based techniques are integrated and are improved using local region-based threshold probing.

In this paper we propose a novel vessel segmentation method that includes a multiscale analysis scheme using Gabor filters and scale production, and a threshold probing technique utilizing the features of retinal vessel network.. Our method is not only good for detecting large and small vessels concurrently, it is also efficient for denoising and for enhancing the responses of line filters, allowing the detection of vessels with low local contrast.

\section{Multiscale analysis}

\subsection{Traditional line detection methods}

Traditional line detection methods proceed either by finding parallel edges [9] [10] or by finding ridges [11] [12] [13]. Parallel edge detection makes use of a barshaped model of lines. In [9], a pair of edge detectors was used to detect the left and right edges of a line:

$$
\begin{aligned}
& E_{l}(x)=-G_{\sigma}^{\prime}\left(x+\frac{w}{2}\right) \\
& E_{r}(x)=G_{\sigma}^{\prime}\left(x-\frac{w}{2}\right)
\end{aligned}
$$

where $\mathrm{w}$ is the width of the line, $G_{\sigma}^{\prime}(x)$ is the first derivative of Gaussian and $\sigma$ is the standard derivation. The responses of $E_{l}$ and $E_{r}$ are then nonlinearly combined to give the line response.

Ridge-based detection methods usually use Gaussianshaped line model. In [11], the ridges are defined as the points on the image where the curvature is the maximum. The second derivate of Gaussian $G_{\sigma}^{\prime \prime}(x)$ is often used as a line detector.

One very important factor in line detection is the scale of the filter. To generate a single maximum response on the center of a line, the widths of the filter and the line 
should be constrained in a proper ratio. For example, the methods described above have to hold

$$
\sigma \geq \frac{w}{\sqrt{3}}
$$

In order to detect lines of arbitrary widths, it is often necessary to iterate the detection procedure in the scale space. Conventionally, all lines in an image [1] are detected using a single optimal value of $\sigma$ (big enough but not too big). Alternatively, the line is emphasized [9] by using the maximum response of all scales. Yet these methods are not entirely satisfactory. Figure 1 summarizes the variances of filter responses to lines and noises along scale space.

First, because excessively wide filters will filter out fine details (small vessels) and the vessels in retinal images can vary from very large to very small, it is rarely possible to find one single filter width value suitable for detecting all vessels.

Further, if the maximum response of all scales is selected, both the large and the small vessels can be emphasized but so too is the associated noise. We propose a novel method that takes these facts into account. Our method includes a multiscale analytical scheme using Gabor filters and scale production, and an adaptive thresholding scheme using adaptive tracking and morphological filtering. Our method is good for detecting large and small vessels concurrently. It also offers an efficient way to denoise and enhance the responses of line filters, allowing the detection of vessels with low local contrast.

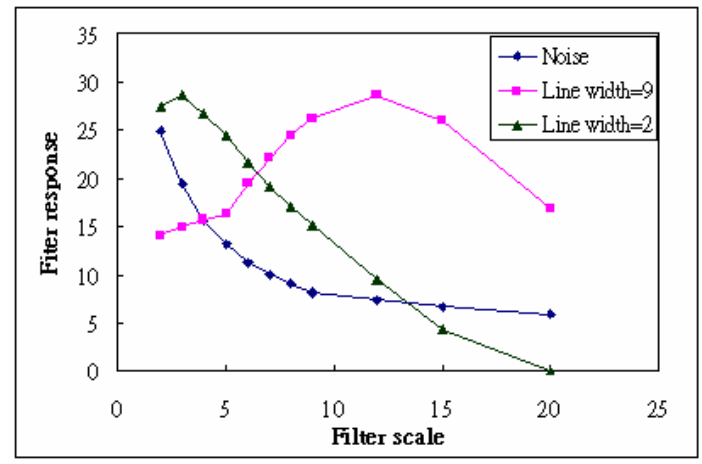

Figure 1 Variances of responses along scale space

\subsection{Vessel detection and noise reduction}

In [14], Mallat illustrated mathematically that signals and noise have different singularities and that edge structures present observable magnitudes along the scales, while noise decreases rapidly. With this observation, we responded to those problems of edge detection and noise reduction by thresholding the multiscale products [15] [16].

Figure 2 shows the effects of scale multiplication. $f$ is a signal corrupted by Gaussian white noise; $W_{s} f$ is the filter response at scale $s ; P_{s} f$ is the product at two adjacent scales. Scale multiplication enhances the edges and filters noise.

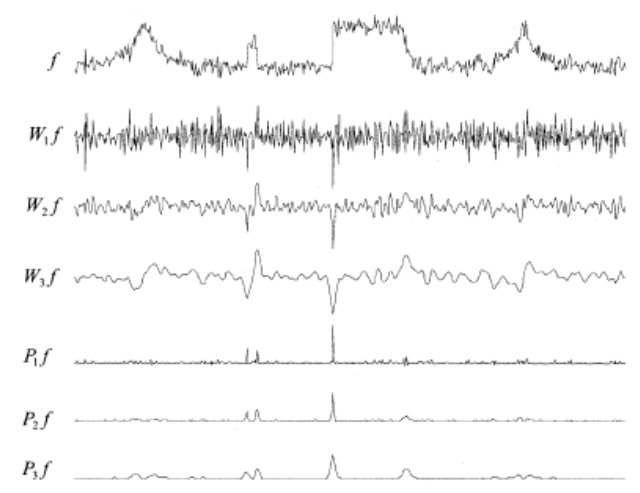

Figure 2. Multiscale edge detection and scale multiplication

Adapting this idea to vessel detection, our multiscale analytical scheme is implemented as follows. First, a proper line detector is produced to obtain the line responses at different scales. We use a Gabor filter [17] here because it is capable of tuning in to specific frequencies and orientations.

In most cases, only the real part of the Gabor filter is used for convolution with the modulation axis parallel to the envelope axis, which is expressed by

$$
\begin{gathered}
g_{\phi}(x, y)=\exp \left\{-\pi\left(\frac{x^{\prime 2}}{\sigma_{x}^{2}}+\frac{y^{\prime 2}}{\sigma_{y}^{2}}\right)\right\} \cos \left(2 \pi f x^{\prime}\right) \\
x^{\prime}=x \cos \phi+y \sin \phi \\
y^{\prime}=-x \sin \phi+y \cos \phi
\end{gathered}
$$

where $\phi$ is the filter direction, $\sigma$ is standard deviation of Gaussian, and $f$ is the frequency of cosine wave. (For convenience, the modulating Gaussians of filters are set to have the same direction as the complex sine grating.)

For multiscale analysis, a scale parameter is added to equation (4) to control the filter size.

$$
g_{\phi, s}(x, y)=\exp \left\{-\pi\left(\frac{x^{\prime 2}}{\left(s \sigma_{x}\right)^{2}}+\frac{y^{\prime 2}}{\left(s \sigma_{y}\right)^{2}}\right)\right\} \cos \left(2 \pi f x^{\prime}\right)
$$

To produce a single peak response on the center of a line of width $w$ using Gabor filters rotated in $n$ directions, Liu [18] has proved that the parameters can be set as follows

$$
\begin{gathered}
f=\beta / w \\
\sigma_{x}=\frac{n \lambda w}{\alpha \beta \pi} \\
\sigma_{y}=\kappa \sigma_{x}
\end{gathered}
$$


where $\alpha \in[1,1.5], \beta \in[0.5,1], \lambda=\sqrt{2 \ln 2 / \pi}$, and $\kappa=0.85$.

Adapting these to multiscale analysis, we modified equation (7) to

$$
g_{\phi, s}(x, y)=\exp \left\{-\pi\left(\frac{x^{\prime 2}}{s^{2}}+\frac{y^{\prime 2}}{(\kappa s)^{2}}\right)\right\} \cos \left(2 \pi f x^{\prime}\right)
$$

And

$$
f=\frac{n \lambda}{\alpha \pi s}
$$

Then, multiscale Gabor filtering can be applied in different directions with the optimal selection of $s$. Figure 3 shows a family of above Gabor filters and the spatial frequency responses.
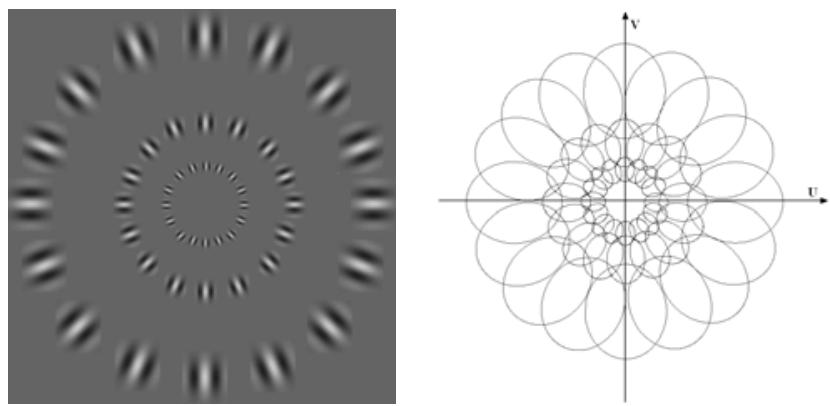

Figure 3 A family of gabor filters in spatial plane and their coverage in spatial frequency plane

The response of Gabor filter can be expressed by

$$
R_{g}(x, y)=g_{\phi, s}(x, y) * f(x, y)
$$

where $f(x, y)$ is the image and $*$ is convolution.

The scale multiplication is defined as the product of Gabor filter responses at two adjacent scales

$$
P^{s_{j}}(x, y)=R_{g}^{s_{j}}(x, y) \cdot R_{g}^{s_{j+1}}(x, y)
$$

where $s_{j}=2^{j}, j=0,1, \ldots, n$.

\section{Threshold probing}

After vessel pixels are emphasized by Gabor filtering, they must be classified as vessel or non-vessel. Hoover [8] applied local region-based threshold probing, which uses line tracking technique to guide the selection of threshold. This technique achieves good performance because the features of retinal vessel network are utilized to help the tracking. Here, we adapt this technique to our Multiscale analysis.

\section{Experimental Results}

We tested our automated vessel segmentation method using the STARE database [1]. Figure 4 shows the effects of scale multiplication. The first row is the original retinal images in STARE database; the second row is the Gabor filter response at small scale; the third row is the Gabor filter response at large scale; the last row is the scale multiplication. We can see that the scale multiplication produces sharper vessel responses and better suppressed noise. Figure 5 compares our method with STARE [8] at very low false positive rate. The first row is the handlabeled images as ground truth; the third row is the segmentation results by [8]; the last row is our method. We can see that a number of small vessels missed by STARE are detected by our method. Note also that some vessels recorded as broken by [8] are connected when our method is used. Figure 6 summarizes the system performance using ROC curve.
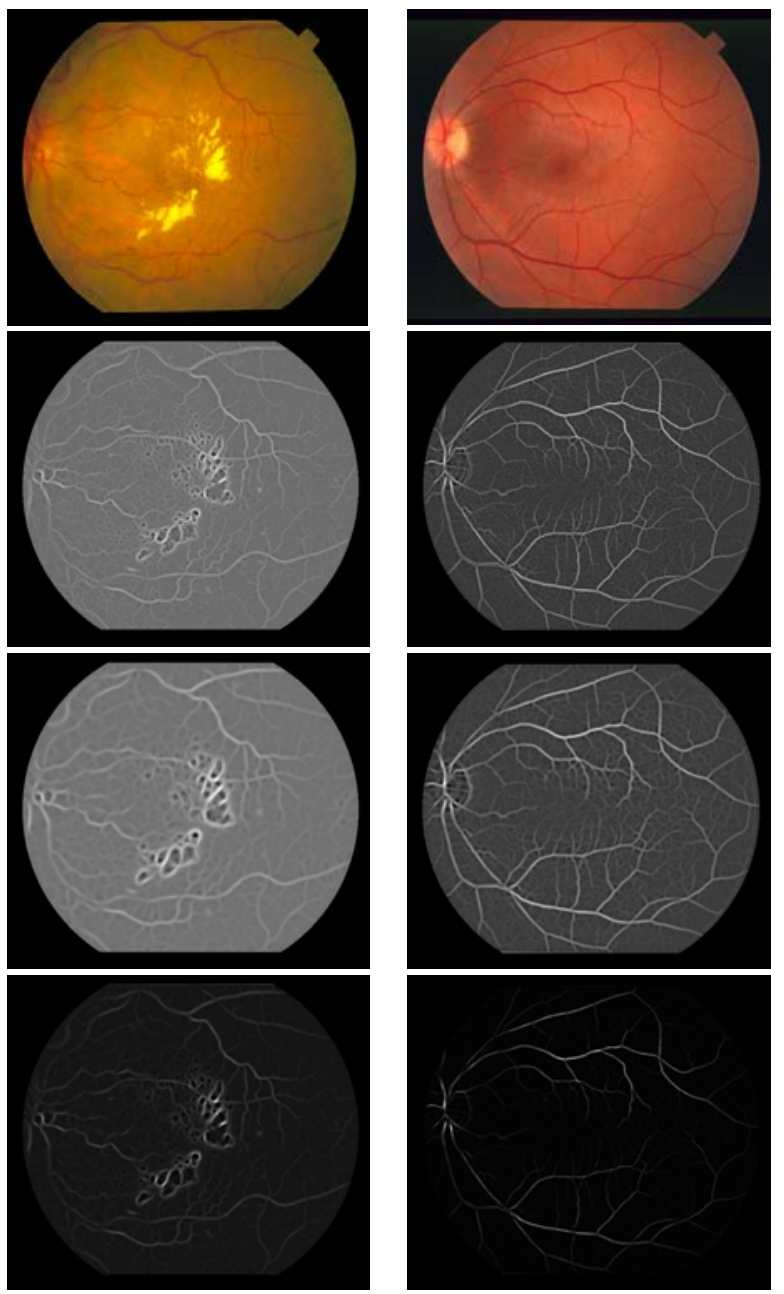

Figure 4. Scale multiplication

\section{Conclusion and Further Works}

We described a method for automated retinal vessel segmentation. Our method includes a multiscale analytical scheme that uses Gabor filters and scale production, and a threshold probing technique. The experimental results demonstrated that our method is good for detecting large 
and small vessels concurrently and also efficiently denoises and enhances the responses of line filters. Line detection is more complicated than edge detection because line widths can vary greatly, depressing the quality of scale multiplication. Further work is required in this area. And, the emphasis of lesions as well as vessels in abnormal retinal images is also an issue that must be addressed.

\section{References}

[1] S. Chaudhuri, S. Chatterjee, N. Katz, M. Nelson, and M. Goldbaum, "Detection of blood vessels in retinal images using two-dimensional matched filters," IEEE Trans. Med. Imag., vol. 8, pp. 263-269, Sept. 1989.

[2] T. Pappas and J. Lim, "A new method for estimation of coronary artery dimensions in angiograms," IEEE Trans. Acoust., Speech, Signal Processing, vol. 36, pp. 1501-1513, Sept. 1988.

[3] Pinz, S. Bernogger, P. Datlinger, and A. Kruger, "Mapping the human retina," IEEE Trans. Med. Imag., vol. 17, pp. 606-619, Aug. 1998.

[4] Y. Sun, "Automated identification of vessel contours in coronary arteriograms by an adaptive tracking algorithm," IEEE Trans. Med. Imag., vol. 8, pp. 78-88, Mar. 1989.

[5] S. Tamura, Y. Okamoto, and K. Yanashima, "Zero-crossing interval correction in tracing eye-fundus blood vessels," Pattern Recognit., vol. 21, no. 3, pp. 227-233, 1988.

[6] Y. Tolias and S. Panas, "A fuzzy vessel tracking algorithm for retinal images based on fuzzy clustering," IEEE Trans. Med. Imag., vol. 17, pp. 263-273, Apr. 1998.

[7] H. Shen, B. Roysam, C.V. Stewart, J.N. Turner, and H.L. Tanenbaum, "Optimal scheduling of tracing computations for real-time vascular landmark extraction from retinal fundus images", IEEE Transactions on Information Technology for Biomedicine, vol. 5, no. 1, pp 77-91, 2001

[8] A. Hoover, V. Kouznetsova, and M. Goldbaum, "Locating blood vessels in retinal images by piecewise threshold probing of a matched filter response," IEEE Trans. on Medical Imaging, vol. 19, no. 3, pp. 203-210, 2000.

[9] T.M. Koller, G. Gerig, G. Székely, and D. Dettwiler, "Multiscale Detection of Curvilinear Structures in 2-D and 3-D Image Data," Fifth Int'l Conf. Computer Vision, pp. 864-869. Los Alamitos, Calif: IEEE CS Press, 1995.

[10] J.B. Subirana-Vilanova and K.K. Sung, "Multi-Scale Vector-Ridge- Detection for Perceptual Organization Without Edges," A.I. Memo 1318, MIT Artificial Intelligence Lab., Cambridge, Mass., Dec. 1992.

[11] J.B.A. Maintz, P.A. van den Elsen, and M.A. Viergever, "Evaluation of Ridge Seeking Operators for Multimodality Medical Image Matching," IEEE Trans. Pattern Analysis and Machine Intelligence, vol. 18, no. 4, pp. 353-365, Apr. 1996.

[12] L. Wang and T. Pavlidis, "Direct Gray-Scale Extraction of Features for Character Recognition," IEEE Trans. Pattern Analysis and Machine Intelligence, vol. 15, no. 10, pp. 1,053-1,067, Oct. 1993.

[13] L.A. Iverson and S.W. Zucker, "Logical/Linear Operators for Image Curves," IEEE Trans. Pattern Analysis and Machine Intelligence, vol. 17, no. 10, pp. 982-996, Oct.
1995.

[14] S. Mallat and S. Zhong, "Characterization of signals from multiscale edges," IEEE Trans. Pattern Anal. Machine Intell., vol. 14, pp. 710-732, July 1992.

[15] P. Bao and L. Zhang, "Noise Reduction for Magnetic Resonance Image via Adaptive Multiscale Products Thresholding," IEEE Trans. Medical Imaging, vol. 22, pp. 1089-1099, Sept. 2003.

[16] P. Bao, L. Zhang, and X. L. Wu, "Canny Edge Detection Enhancement by Scale Multiplication", IEEE Trans. Pattern Analysis and Machine Intelligence, Vol. 27, No. 9, Sept. 2005

[17] J.G. Daugman, "Uncertainty relation for resolution in space, spatial frequency, and orientation optimized by twodimensional visual cortical filters," Journal of the Optical Society of America A, vol. 2, pp. 1160-1169, 1985.

[18] Z.-Q. Liu, J. Cai, and R. Buse, Handwriting Recognition: Soft Computing and Probabilistic Approaches, pp. 31-57, Springer, Berlin, 2003
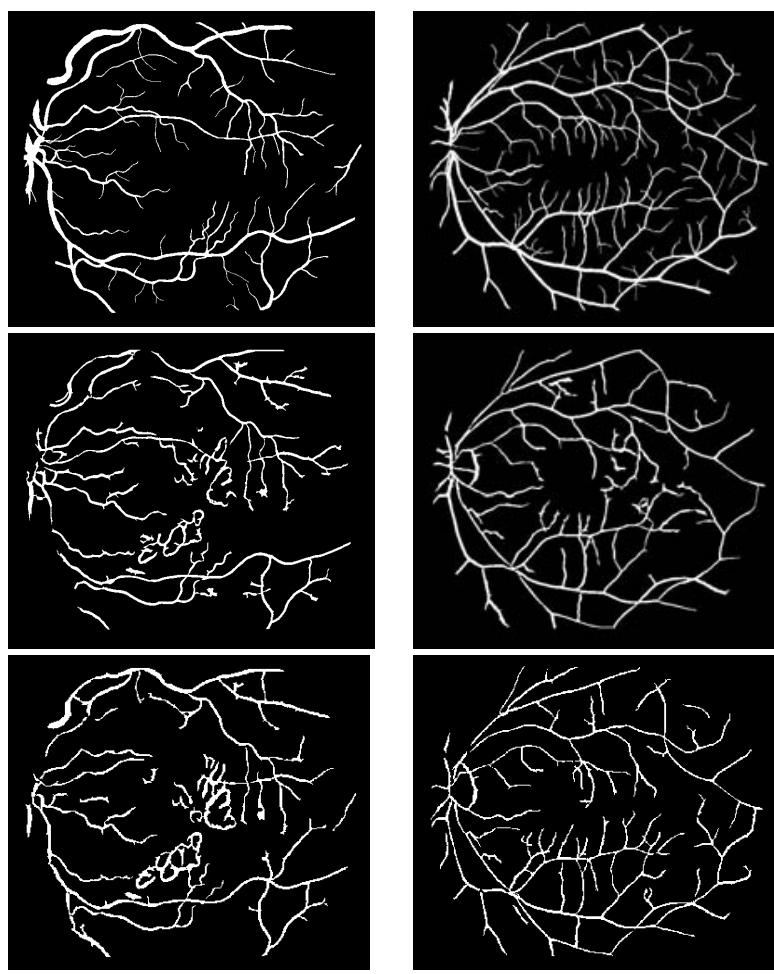

Figure 5. Segmentation results

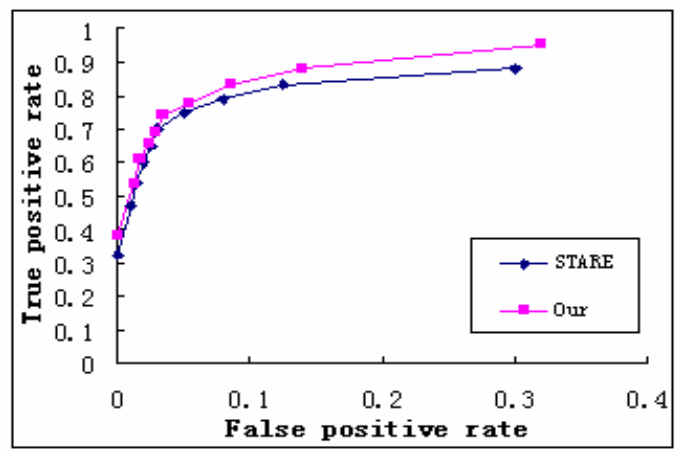

Figure 6. System evaluation using ROC 